Main Outcome Measurements Sex, limb dominance, joint angles and excursions, and muscle excitation amplitudes during the flight phase of the drop-jumps were extracted. These variables were normalized, reduced and submitted to a logistic regression.

Results Twenty-nine variables were reduced to a seven variable logistic regression model that included trunk and pelvis lateral tilt, pelvis internal rotation, hip abduction, trunk and pelvis joint excursion, and biceps femoris muscle excitation. Using these variable the model correctly classified $74 \%$ of the landings. The same variables are present for both males and females.

Conclusions Regardless of sex, an athlete who presents the identified movement and control patterns during the flight phase risks a failed landing, potentially increasing the risk of injury. Typical prophylactic interventions focus on landing characteristics. This research indicates for the first time that modifying what occurs prior to landing is critical and must be addressed through training.

\section{THE PREVENTIVE EFFECT OF TARGETED ADDUCTOR TRAINING ON GROIN PAIN FROM FOOTBALL PLAYERS}

Julia Smakal, Nadja Jamrog, Bartosz Wojanowski. Privatpraxis Orthopädie, Dortmund, Germany

\subsection{6/bjsports-2021-IOC.50}

Background Groin pain is a widespread problem in football. Not only in the professional leagues, even the amateur and the youth sector have to handle it. Consistently, there are training and game absenteeism due to existing pain in this area.

Objective The aim was to describe the preventive effect of a separate training of the hip joint adductors on groin pain in order to reduce their prevalence.

Design Randomized prospective intervention study.

Setting Twelve amateur football teams were grouped and supervised during a period of six months (2 preparation, 4 first half of the season).

Patients (or Participants) Twelve amateur football teams participated (6 teams, 121 players, aged $25 \pm 8$ yrs, in the intervention group, 6 teams, 115 players, aged $26 \pm 5 \mathrm{yrs}$, in the control group)

Interventions (or Assessment of Risk Factors) The intervention group worked on three targeted exercises to strengthen the adductors, performed said exercise three times a week during their preiod and once a week during their season ( $3 \times 15$ reps, 30'). The content included stabilization exercises with their own body weight and a 'Doiserband' as an additive.

Main Outcome Measurements The prevalence of groin pain was recorded weekly over a period of 16 weeks during the competition phase per VAS and a short questionnaire. The weekly activity of each player was also recorded.

Results The average prevalence of groin pain was $12.5 \%$ (95\% CI. $11,3 \%$ to $13,7 \%)$ in the intervention group and $20.3 \%(95 \% \mathrm{Cl} .29,0 \%$ bis $21,6 \%)$ in the control group. This result is a $41 \%$ lower risk for the occurrence of groin pain, if separate exercises for strengthening would be performed additionally.
Conclusions It becomes apparent that a strengthening training of the adductors can reduces the prevalence of groin pain in the observed sample. Therefore, a targeted and regular training program is recommended to strengthen the adductors, and to preventively reduce the risk of training and competition failures.

\section{SUPERVISED IMPLEMENTATION OF A NEUROMUSCULAR TRAINING WARM-UP PROGRAMME TO IMPROVE ADHERENCE AND REDUCE INJURIES IN YOUTH BASKETBALL: A CLUSTER RANDOMISED TRIAL}

1,2,30luwatoyosi Owoeye, ${ }^{2,3,4,7}$ Kati Pasanen, ${ }^{2}$ Anu Raisanen, ${ }^{2}$ Kimberley Befus, ${ }^{2}$ Tyler J Tait, ${ }^{2}$ Carlyn Stilling, ${ }^{2}$ Vineetha Warriyar, ${ }^{2,5}$ Luz Palacios-Derflingher, $2,3,5,6,7,8$ Carolyn Emery. ${ }^{1}$ Department of Physical Therapy and Athletic Training, Doisy College of Health Sciences, Saint Louis University, Missouri, USA; ${ }^{2}$ Sport Injury Prevention Research Centre, Faculty of Kinesiology, University of Calgary, Calgary, Canada; ${ }^{3}$ Alberta Children's Hospital Research Institute, University of Calgary, Calgary, Canada; ${ }^{4}$ Tampere Research Center of Sports Medicine, UKK Institute, Tampere, Finland; ${ }^{5}$ Department of Community Health Sciences, Cumming School of Medicine, University of Calgary, Calgary, Canada; ${ }^{6}$ Department of Pediatrics, Cumming School of Medicine, University of Calgary, Calgary, Canada; ${ }^{7}$ McCaig Institute for Bone and Joint Health, University of Calgary, Calgary, Canada; ${ }^{8} \mathrm{O}^{\prime}$ Brien Institute for Public Health, University of Calgary, Calgary, Canada

\subsection{6/bjsports-2021-IOC.51}

Background The efficacy of neuromuscular training (NMT) programmes has been extensively documented; however, little is known about the best strategies to translate them into practice.

Objective To compare the effects of a supervised vs. unsupervised implementation of an NMT warm-up programme on team adherence and injury risk in youth basketball players.

Design A pragmatic cluster randomised controlled trial.

Setting High school basketball teams in Alberta, Canada.

Participants 31 teams (18 female teams) comprising 307 players (age range: 14-18 years).

Interventions A structured pre-season coach workshop with (intervention) or without (control) weekly research staff supervision of a 10-minute NMT warm-up programme, comprising 13 exercises was administered. Teams were asked to perform the NMT warm-up programme before every practice and game through the 2017/2018 basketball season.

Main Outcome Measurements Team adherence, evaluated as cumulative utilisation (proportion of total NMT sessions possible), utilisation fidelity (average number of exercises completed per NMT session) and utilisation frequency (average number of NMT sessions completed per week), was tracked daily by team designates. All-complaint injuries were collected weekly. Wilcoxon sign rank tests or Poisson regressions were used for the analyses, with Bonferroni corrections.

Results No significant differences were found in the median (range) cumulative utilisation $[80 \% \quad(32 \%-100 \%)$ vs. $75 \%$ (16\%-100\%)], utilisation fidelity $[12.1 \quad(5.5-13.0)$ vs. 11.4 $(5.1-13.0)]$ and utilisation frequency $[2.2(0.9-4.1)$ vs. 2.2 $(0.5-4.7)]$ between intervention and control groups, respectively (all $\mathrm{p}>0.017$ ). Injury incidence rates, adjusted for cluster by team, sex and age did not differ by groups for all injuries [incidence rate ratios (IRR) $=1.21$ (97.5\%CI: 0.73-1.99)] and lower extremity injuries [IRR $=1.10$ (97.5\%CI: $0.73-$ 1.66)].

Conclusions No additional benefits were found in adherence or injury risk reduction following a supervised implementation 